\title{
METAS EDUCATIVAS 2021. LA EDUCACIÓN TÉCNICA PARA EL DESARROLLO SOCIAL EN EL PARAGUAY
}

\section{EDUCATIONAL GOALS 2021. TECHNICAL EDUCATION FOR SOCIAL DEVELOPMENT IN PARAGUAY}

\author{
Daxi Duarte de García
}

\begin{abstract}
The objective of the study is to contribute to the reflection on the level of achievement that the States signatories of the project have reached in relation to the 11 educational goals proposed by the Educational Goals Project 2021, three years after the end of the period of its development; and analyze the progress made in Paraguay of the goal of the Sixth Educational Goals 2021, that of Favoring the connection between education and employment through technical-professional education (ETP). The work is part of a review and documentary analysis, based on a collection of bibliographic and electronic sources from different authors and multilateral organizations, specifically in the publications of the qualitative and quantitative indicators of Miradas sobre la Educación en Iberoamérica: Advance in Educational Goals 2021, in the years 2014, 2016 and 2018 respectively and other compilations published locally. Data was taken from different entities to contrast the information and finally propose the strengthening of Paraguayan education to achieve a greater professional technical capacity in the national population, to respond to the labor demand of the industrial sector, but aware that a development Economic development must go hand in hand with social development based on education, the only way of social change.
\end{abstract}

Key words: education, technical education, labor access, social development, results

\section{RESUMEN}

El objetivo del estudio es contribuir a la reflexión sobre el nivel de logro que han alcanzado los Estados firmantes del proyecto en torno a las 11 metas educativas propuestas por el Proyecto Metas Educativas 2021, a tres años vista de la finalización del periodo de su desarrollo; y analizar el avance logrado en el Paraguay del objetivo de la Sexta Metas Educativas 2021, la de Favorecer la conexión entre la educación y el empleo a través de la educación técnico-profesional (ETP). El trabajo se enmarca en una revisión y análisis documental, basada en un arqueo de fuentes bibliográficas y electrónicas de diferentes autores y organismos multilaterales, específicamente en las publicaciones de los indicadores cualitativos y cuantitativos de Miradas sobre la Educación en Iberoamérica: Avance en las Metas Educativas 2021, en los años 2014, 2016 y 2018 respectivamente y otras recopilaciones publicadas a nivel local. Se tomó datos de distintas entidades para contrastar las informaciones y finalmente proponer el fortalecimiento de la educación paraguaya para el logro de una mayor capacidad técnico profesional en la población nacional, para dar respuesta a la demanda laboral del sector industrial, pero consciente de que un desarrollo económico debe ir paralelo al desarrollo social basado en la educación, única vía de un cambio social.

Palabras clave: educación, educación técnica, acceso laboral, desarrollo social, resultados 
Fecha de recepción: 9 de noviembre de 2018.

Fecha de aceptación: 12 de diciembre de 2018. 


\section{INTRODUCCIÓN}

La educación ha subsistido en el tiempo y en el espacio, ha pasado por múltiples cambios que gravitan al desarrollo de un sistema que emplea un procedimiento determinado para divulgar ideas entre los estudiantes, que nuclean las piezas más básicas de una familia y de la sociedad. En tanto el tipo de formación impartida ya no se ajusta con las nuevas tecnologías del presente. Obliga articular una fuerte innovación en este factor tan transcendental, en la vida de las personas y de su fuerte contribución para el desarrollo de un país. Desde el origen de la humanidad, se ha reconocido de un modo sustantivo el conocimiento y el aprendizaje. "Si un hombre deja de lado la educación, camina cojo hasta el final de su vida, subrayó Platón” (Baelo, Fernández y Valle, 2018, p.8).

Tedesco (2016), refiere que al "analizar los avances y desafíos de la educación en América Latina provoca sentimientos ambiguos", y que con ella:

"no se puede evitar la frustración ante la lentitud de los progresos y los riesgos de retrocesos. Por otro lado, en cambio, el dinamismo de las sociedades siempre abre expectativas de promover los cambios tan reiteradamente anhelados. Que, desde el punto de vista político es importante asociar ambos sentimientos. Para que las expectativas de cambios no sean una mera ingenuidad es necesario aprender de las frustraciones, reconocer el agotamiento de algunos esquemas de interpretación utilizados hasta ahora e intentar formular hipótesis que permitan superar la pérdida de fertilidad de las ideas conocidas".

De la misma forma el autor sigue expresando que:

"América Latina se distingue por ser una región donde se expande la cobertura escolar; los alumnos permanecen en la escuela una cantidad significativa de años, pero no logran resultados satisfactorios de aprendizaje. El fracaso escolar constituye el núcleo duro del problema educativo latinoamericano. En contextos donde existen recursos financieros y voluntad política para garantizar el derecho a una educación de calidad para todos, es necesario enfrentar la renovación del saber pedagógico que permita resolver los problemas de aprendizaje que presentan los estudiantes. Dicha renovación es un factor crucial para promover los cambios necesarios en la formación docente". (Tedesco, 2016, p. 7).

En el Paraguay, una vez producida la caída de la dictadura de Stroessner en 1989, se inicia una reforma de la educación como un imperativo para la construcción de una sociedad democrática. En ese momento el Estado promovió una serie de iniciativas en el ámbito educativo: la creación en 1990 del Consejo Asesor de la Reforma Educativa (CARE) con el objetivo de formular una reforma integral del sistema educacional paraguayo, previa elaboración de un diagnóstico de la situación general, de tal forma a plantear propuestas y alternativas que puedan solucionar las deficiencias del sistema (CARE, 1992); la realización entre 1992 y 1993 de dos congresos educativos nacionales y 19 regionales convocados por el Parlamento con la participación de docentes y otros actores educativos y en los que se discutieron temas tales como: los principios y filosofía de la reforma educativa, bilingüismo, formación docente, administración educativa, educación indígena, entre otros (MEC, 2000); la formulación de una ley general que redefinió el marco normativo e institucional del sistema educativo (MEC, 1998) y la introducción progresiva de nuevos programas de estudios en todos los niveles educativos. (Molinas, Elías y Vera, 2004).

El proyecto "Metas Educativas 2021: la educación que queremos para la generación de los Bicentenarios" es una iniciativa que han puesto en marcha los países iberoamericanos. La finalidad es mejorar la calidad y la equidad en la educación para hacer frente a la pobreza y a la desigualdad y favorecer la inclusión social. Las Metas Educativas 2021 se han planteado en cuatro niveles: metas 
generales, metas específicas, indicadores y niveles de logro. Con el fin de concretar el cambio esperado en cada una de las metas específicas, se han establecido indicadores (38), que expresan el criterio que será utilizado para evaluar los avances en las metas, así como niveles de logro, que supone la aplicación del indicador elegido a las diferentes metas específicas y que expresan el objetivo que se espera alcancen los países en 2021 (OEI, 2011).

Las Metas Educativas 2021, constituyen un nuevo marco internacional (regional) que ha tenido influencia en las políticas educativas paraguayas. La Organización de Estados Iberoamericanos (OEI) ha tenido una creciente presencia en el país, en particular a partir del año 2007 y ha promovido la incorporación de los objetivos de las Metas en las políticas educativas nacionales.

En 2008 los ministros de educación de los países de Iberoamérica aprobaron el compromiso de preparar la propuesta "Metas Educativas 2021: la educación que queremos para la generación de los Bicentenarios", lo que significó la elaboración de objetivos, metas y mecanismos de evaluación regional, vinculado a los planes nacionales, e iniciar un proceso de reflexión para dotar a la propuesta de un fondo estructural y solidario. El "Congreso Iberoamericano de Educación Metas 2021", que tiene tuvo lugar en Buenos Aires en septiembre de 2010, constituye la culminación de encuentros y debates en los que se fue ajustando la integración de los diferentes países a este proyecto regional, a partir de identificar los rasgos específicos que caracterizan a cada uno de ellos (SITEAL, 2010).

Los objetivos del trabajo son: contribuir a la reflexión sobre el nivel de logro que han alcanzado los Estados firmantes del proyecto en torno a las 11 metas educativas propuestas a tres años vista de la finalización del periodo de su desarrollo; analizar el avance logrado en el Paraguay del objetivo de la Sexta Metas Educativas 2021, la de favorecer la conexión entre la educación y el empleo a través de la educación técnico-profesional (ETP). Se enmarca en una revisión y análisis documental, basada en un arqueo de fuentes bibliográficas y electrónicas de diferentes autores y organismos multilaterales.

Vickery (1970) señaló que:

"los métodos de recuperación, entre los que se cuenta el análisis documental, responden a tres necesidades informativas de los usuarios, en primer lugar, conocer lo que otros pares científicos han hecho o están realizando en un campo específico; en segundo lugar, conocer segmentos específicos de información de algún documento en particular; y por último, conocer la totalidad de información relevante que exista sobre un tema específico". (p.154).

Fox (2005) "utiliza la expresión: «tratamiento documental», dentro de la cual incluye al análisis que se aplica a los documentos cuando entran a formar parte de un fondo documental, y cuya finalidad es elaborar una nueva representación de su contenido para facilitar su consulta." (p.21) Por tanto, la revisión y análisis documental permite realizar exploraciones retrospectivas y recuperar el documento que se requiere en el momento en que se precise utilizar, comparar, es decir que se puede identificar y localizar cualquier documento, así como conocer su contenido. 


\section{META EDUCATIVA 6: FAVORECER LA CONEXIÓN ENTRE LA EDUCACIÓN Y EL EMPLEO A TRAVÉS DE LA EDUCACIÓN TÉCNICO-PROFESIONAL (ETP)}

Terminada la segunda guerra mundial, se inicia el auge en torno a la educación técnica, que ha originado nuevos y diversos roles en los métodos de desarrollo económico y productivo de los países. En este sentido, la formación técnica ha asumido un papel fundamental en los entornos educativos en la ejecución de políticas de desarrollo social.

La educación secundaria latinoamericana históricamente se ha basado en dos modelos institucionales: la educación secundaria académica y/o general (ESA), orientada a la continuidad de estudios superiores, basada en contenidos de fundamento sin pretensiones de una directa relación con el mundo del trabajo; y la educación técnica y comercial (EST), centrada más específicamente a la inserción laboral inmediata. Desde hace un par de décadas en el marco de profundas transformaciones estos dos modelos paralelos han comenzado a debatirse (Jacinto, 2009, citado por Del Giorgio Solfa, Sierra, Vescio, \& Fernández Zocco, 2018, p. 195)

En, Vidales Calderón (2013) se destaca que es de:

"Vital importancia la generación de técnicos especializados para aquellos países en transición hacia
una sociedad más basada en la innovación. Paralelamente, se suma a la importancia de la educación
técnica, que el período de formación de la educación media coincide con un periodo en la vida de los
escolarizados donde buscan modelos a seguir. En general se analizan tres momentos importantes en
el desarrollo de las personas y los modelos que se tienen disponibles en cada uno de ellos: antes de
empezar a realizar una actividad económica, al momento de ingresar a la población económicamente
activa y posterior, cuando ya una persona es asalariada. De los tres, quizás el más importante es el
momento de preparación para iniciar una carrera profesional, etapa en la cual los jóvenes tienen dos
modelos importantes: los maestros y los padres, ambos con una responsabilidad primordial en el
fomento del emprendimiento entre los jóvenes". (p. 14)

En este sentido, es indicado infiltrarse en el enfoque de política pública en el orden nacional en torno a la enseñanza técnico-profesional tema de interés, para atisbar el escenario educativo en el Paraguay; que en efecto es complejo y sumamente sensible.

Del Giorgio Solfa, y otros (2018), publican que solo el 32\% de los jóvenes ha concluido su educación secundaria y el 5\% el nivel terciario. Además, en este grupo se presenta una alta tasa de desempleo e inactividad (Presidencia de la República de Paraguay, 2011). Como así también el Estado afirma en el documento de Plan Nacional para el Mejoramiento de la Educación Técnica y Profesional en el Paraguay 2011 -2013, que:

"Los programas de estudio no cuentan con mecanismos que aseguren inserción laboral segura y de calidad, y como consecuencia de estas deficiencias se presenta la deserción y el abandono escolar. Esto es acompañado de una complicada situación en el mercado laboral que muestra una temprana incorporación de jóvenes desde los 10 años, concentración de los ingresos del país y poco desarrollo industrial, mayoritariamente rural con escaso o nulo acceso a la tecnología y a créditos de desarrollo. Por otro lado, desde el sector industrial se afirma que hay una gran escasez de mano de obra capacitada para ocupar los espacios requeridos por mercado laboral productivo" (Messina et al., 1996; Ministerio de Educación y Cultura, 2008; Ramírez \& González, 2010; Rivero, 2008 citado por Del Giorgio Solfa, Sierra, Vescio, \& Fernández Zocco, 2018, p. 190). 
Los autores, señalan que, si bien se han desarrollado una vasta cantidad de programas, estos no han producido grandes mejoras; podríamos mencionar dos grandes problemáticas en relación a esto. Muchos de los proyectos desarrollados no contaban con un estudio previo de la región en la que se implementaría, por lo que los contenidos poco tenían que ver con las demandas laborales del territorio, y, en segundo lugar, las experiencias aisladas se han visto sumamente desarticuladas. Frente a esta crítica situación, entre el año 2004 y el 2009 el Ministerio de Educación y Cultura de la Nación ha generado programas formales y no formales de educación para el trabajo formando profesionales capacitados en un área específica acorde a la zona donde reside, y acompañando las políticas de mejora, el Ministerio de Justicia y Trabajo programas de iniciación y capacitación laboral desde el Servicio Nacional de Promoción Profesional (SNPP) y desde el Sistema Nacional de Formación y Capacitación Laboral (SINAFOCAL). (Ministerio de Educación y Ciencias, 2011) Además, en el área de formación técnica se dispone de distintas ofertas formativas, la Iniciación Profesional Agropecuaria en el nivel básico, los bachilleratos Técnicos y la Formación Profesional en el nivel medio, las Tecnicaturas del nivel superior y los programas. Cabe destacar que la definición dada a la formación profesional es la de generar vinculación con el sector de la producción y detectar las necesidades de capacitación mediante alianzas estratégica con estamentos locales y las mesas de trabajo sobre empleabilidad (Ministerio de Educación y Cultura, 2008).

Uno de los programas más mencionados es el Vulcano, que comprende la Formación Profesional inicial, destinado a la población egresada del programa de Educación básica para jóvenes y adultos del Paraguay PRODEPA equivalente a la educación básica. Vulcano tiene como fin potenciar la orientación laboral y desarrollar una formación basada en competencias. Este modelo se refiere al desarrollo de capacidades y actitudes con un perfil definido para el ámbito laboral, la participación de los representantes del área productiva y laboral, la formación desde distintos enfoques: empresarial y psicológico (Messina et al., 1996; Ministerio de Educación y Cultura, 2008; Ramírez \& González, 2010; Rivero, 2008 citado por Del Giorgio Solfa, Sierra, Vescio, \& Fernández Zocco, 2018, p. 195).

Desde estos antecedentes es muy apropiado realizar una mirada a la conexión entre la educación y el empleo en el Paraguay. En ese sentido es importante iniciar el abordaje desde el año 2010, cuando el gobierno de turno dio amplia difusión e impulso al proyecto Metas Educativas 2021: la educación que queremos para la generación de los Bicentenarios, cuyos objetivos son absolutamente coherentes, ocurrentes y necesario para lograr una transformación social de cara al ingreso a la historia del siglo XXI.

En razón de que Metas Educativas 2021, se enmarca en el logro de una educación más justa, con mayor equidad social y mayor nivel cultural, anhelo que involucra a toda la ciudadanía iberoamericana; que, por cierto, es muy bien visto por los jefes de estados, incluido el de Paraguay. Esto indudablemente ha provocado una gran expectativa en los ciudadanos que conciben en la capacidad transformadora de la educación y con ella honrar la deuda histórica vigente con los seres humanos. Cuyos objetivos son ambiciosos, de gran impacto en la comunidad académica, pero de poca repercusión en términos de políticas públicas en el Paraguay. 


\title{
2. ANTECEDENTES DE LA FORMACIÓN Y EDUCACIÓN TÉCNICO PROFESIONAL EN EL PARAGUAY
}

Una publicación del Ministerio de Educación y Cultura indica que:

\begin{abstract}
"la educación técnica fue introducida en el sistema educativo como un medio de diversificar la oferta educativa del nivel medio y como un fin en cuanto a la preparación de recursos humanos para los niveles medios, sector desprovisto entonces de personal calificado. Sus componentes curriculares se incorporaron al sistema educativo formal del Ministerio de Educación y Culto del Paraguay en el marco de las Innovaciones Educacionales en 1973, con el objetivo de formar a los jóvenes para su incorporación en oficios y ocupaciones técnicas, formación de mano de obra especializada y técnicos del nivel medio y terciario, orientados hacia logros de mayor productividad acorde al proceso de desarrollo que experimentaba el país". (MEC, 2011, p. 8)
\end{abstract}

De la misma forma dice que actualmente, el MEC dispone de cuatro tipos de ofertas formativas diferenciadas; por una parte, la Iniciación Profesional Agropecuaria que se implementa en el nivel de la Educación Escolar Básica y está dirigida a estudiantes del tercer ciclo, los Bachilleratos Técnicos y la Formación Profesional que se implementan en el nivel de la Educación Media, las tecnicaturas de nivel superior que se implementan en el nivel de la Educación Superior y los programas no formales de formación y capacitación laboral para jóvenes y adultos que se implementan en el ámbito de la Educación Permanente.

En el mismo documento de referencia, el Ministerio de Agricultura y Ganadería (MAG): expone que las primeras Escuelas de Agricultura en Paraguay fueron creadas en el año 1882, y las escuelas agrícolas regionales en el año 1931, aunque recién en 1992 se reconoció a estas instituciones educativas como escuelas agrícolas. En el año 1999 se concreta la firma de un convenio por el cual se adoptan e implementan las nuevas modalidades en instituciones del MEC. La función principal de estas unidades escolares es la formación del profesional de nivel básico, el bachillerato técnico agropecuario de nivel medio, y la capacitación de productores agrícolas jóvenes y adultos, hombres y mujeres, mediante la vinculación de la escuela con su comunidad. También realizan actividades como experimentación y extensión agrícola, en coordinación con las dependencias respectivas; y sumado a ello, las escuelas agrícolas facilitan por una parte la implementación de especializaciones terminales del bachillerato técnico agropecuario, a través de la realización de variados proyectos supervisados, los cuales comprenden el ciclo agrícola en todas sus etapas, y por otra, la introducción del requisito de práctica profesional o pasantías fuera del establecimiento que puede abarcar todas o algunas de las fases productivas. En el año 2004, las modalidades ofrecidas fueron ampliadas con la incorporación de la Iniciación Profesional Agropecuaria (IPA) y la de Técnico en Administración de Finca. (MEC, 2011, p. 9)

En esta triada publicación, también participa el Ministerio de Justicia y Trabajo (MEC, 2011 p 9; expresa que el Sistema Nacional de Promoción Profesional (SNPP) fue creado en 1971 y tiene la finalidad de promover y desarrollar la formación, capacitación y perfeccionamiento de los trabajadores en todos los niveles de la pirámide ocupacional y los diferentes sectores de la economía. Los cursos ofertados por esta entidad se dirigen a mayores de 18 años y son accesibles en cualquier punto del país a través de numerosas sedes. Las ofertas formativas abarcan las áreas agropecuarias, industrial y de servicios. Por otra parte, el Sistema Nacional de Formación y Capacitación Laboral (SINAFOCAL) se crea con el objetivo de prestar a sus beneficiarios oportunidades de capacitación y formación en sus diversas modalidades. 
Y otros programas de capacitación laboral que coadyuva entre las entidades gubernamentales y privadas que cuentan con programas de capacitación laboral se citan: las instituciones educativas militares, el Instituto Nacional de Salud, el Centro de Apoyo a las Empresas (CEPAE), el Instituto Paraguayo de Artesanía (IPA), el Centro de Adiestramiento en Servicio (CAES), la Administración Nacional de Electricidad (ANDE), la Compañía Paraguaya de Comunicaciones (COPACO), la Cámara Paraguaya de la Construcción (CAPACO) y otras. (MEC, 2011, p. 9)

En el Informe Nacional Paraguay Educación para Todos 2000 -2015 (Elías y Briet, (2014, p. 172), los resultados de los programas mencionados precedentemente, se destaca que la tasa de alfabetismo de las personas jóvenes y adultas de 15 a 24 años progresó, llegando al $98 \%$ en pie de igualdad de las mujeres y los hombres; que los niveles educacionales de esta población (15 a 24 años) incrementaron. En 2012, uno de cada 3 jóvenes ha culminado la Educación Secundaria Alta (EM), con una mayor proporción de mujeres, lo que indica que permanecen más tiempo en el sistema Educativo que los hombres. Muy importante por cierto pues las Tasas Brutas de CINE 2 (EEB 3er ciclo o Secundaria Baja) y CINE 3 (EM o Secundaria Alta) han mejorado significativamente y alcanzado respectivamente el $82 \%$ y $68 \%$ en 2012. Sin hacer ninguna mención y o información referente al CINE 5.

"El informe puntualiza que la elevada cantidad de jóvenes rurales y de comunidades indígenas que no disponen de las competencias necesarias para trabajar y ganarse la vida decentemente; que la mayor parte de las personas jóvenes y adultas de 15 a 24 años acude a programas de secundaria general. Sin embargo, se registra un incremento de la matrícula hacia el bachiller técnico profesional en el último decenio; los programas de FP y FPI de la Dirección General de Educación Permanente registran una proporción importante de jóvenes. De la misma forma, en FP, casi 1 de cada 3 de los inscritos tienen menos de 18 años. Apuntan que la tasa de transición ha conocido un incremento notable en este periodo, y alcanza el 97\% en 2011 lo que evidencia la transición más fluida que existe entre estos dos niveles (primario y secundario)". (Elias, Briet, 2014, p. 172)

"Con relación a las dificultades que deben sopesar por la falta de continuidad de acciones implementadas debido a los cambios de gobiernos y autoridades; la falta de actualización del análisis del mercado laboral para diseñar propuestas de educación técnico- profesional en adecuación con la demanda de la sociedad, en términos de bienes y servicios; la deficiencia de recursos humanos capacitados en el ámbito de educación para el trabajo; los recursos financieros insuficientes destinados a talleres y herramientas para educación técnico-profesional y las dificultades de articulación interinstitucional e intersectorial para la implementación de acciones", indica el Informe Nacional Paraguay Educación para Todos 2000 -2015. (Elias, Briet, 2014, p.173)

\section{MARCO LEGAL QUE RIGE EN EL PARAGUAY- EDUCACIÓN TP}

Para considerar un Programa Educativo siempre es significativo contar con un marco jurídico, en ese sentido se enfatiza los artículos contenidos en La Carta Magna y las leyes que rigen en la ETP del CINE 3 y CINE 5, para recrear una mirada más a fondo a fin de sustentar el cumplimiento del mismo. 
Tabla 1: Marco Legal en el Paraguay que rige en la ETP de las áreas CINE 3 y CINE 5

Constitución Nacional
de la República de
Paraguay (1992) La
Constitución Nacional
expresa el derecho a la
educación y la
protección del/del
niño/a.

En el Artículo 76 sobre las Obligaciones del Estado se recalca el carácter obligatorio de la educación escolar básica, y gratuito en las escuelas públicas. Por otra parte, el Estado se compromete en fomentar la enseñanza media, técnica, agropecuaria, industrial y la superior o universitaria, así como la investigación científica y tecnológica. "La organización del sistema educativo es responsabilidad esencial del Estado, con la participación de las distintas comunidades educativas. Este sistema abarca a los sectores públicos y privados, así como al ámbito escolar y extraescolar".

El Artículo 78, sobre la educación técnica recalca que "El Estado

fomentará la capacitación para el trabajo por medio de la enseñanza técnica, a fin de formar los recursos humanos requeridos para el desarrollo nacional"

\section{Ley General de Educación $N^{\circ} 1.264 / 98$}

En la sección IV de la presente ley se delinean las pautas legales sobre educación media, abarcando desde el

Artículo 37 al 42:

Artículo 37, La

Educación Media comprende el bachillerato o la formación profesional y tendrá tres cursos académicos. Busca como objetivos la incorporación activa del alumno a la vida social y al trabajo productivo o su acceso a la educación de nivel superior. El Estado fomentará el acceso a la educación media previniendo los recursos necesarios para ello.

Artículo 38, La educación media orientará a los alumnos en el proceso de su maduración intelectual y afectiva de manera que puedan integrarse crítica y creativamente en su propia cultura, así como adquirir los conocimientos y habilidades que les permitan desempeñar sus compromisos sociales con responsabilidades y competencia. Artículo 39, El Ministerio de

Educación y Cultura establecerá el diseño curricular con los objetivos y el sistema de evaluación propios de esta etapa, que será organizado por áreas.

\begin{tabular}{|l|l}
\hline Ley N N $^{\circ} 264$ Pasantía & Ley N$^{\circ} 5.636$ \\
Educativa Laboral & Pasantía Educativa \\
Artículo $1^{\circ}$. - La & Laboral Técnica \\
presente Ley tiene por & Superior.
\end{tabular}

tiene por objeto regular la Pasantía Educativa Laboral de las especialidades del Bachillerato Técnico de la Educación Media, que realizan los alumnos de las instituciones de enseñanza, sean ellas públicas, privadas o privadas subvencionadas. Artículo $2^{\circ}$. - Se entiende como "Pasantía Educativa Laboral" el acto educativo supervisado por el Ministerio de Educación y Cultura, que desarrolla el alumno en el ámbito de una empresa u organización privada legalmente constituida o en una entidad pública, con la finalidad de complementar el proceso de aprendizaje por medio de la aplicación práctica de la formación académica que recibe.

Artículo 3․ - Las

pasantías educativas laborales podrán ser obligatorias y no obligatorias. Se consideran "obligatorias" aquéllas que forman parte del itinerario formativo prescripto en el currículum de la especialidad y que constituyen un requisito para acceder al título correspondiente. Se entiende como "no obligatorias" cuando no están establecidas como requisito en el plan de estudio del curso respectivo.
Artículo 1. ${ }^{\circ}$ La presente ley tiene por objeto regular la Pasantía Educativa Laboral de las carreras de Formación Técnica Superior de la Educación Superior, que realizan los alumnos de las instituciones de enseñanza, sean ellas públicas, privadas o privadas subvencionadas, con ofertas de tecnicatura oficialmente habilitada por el Ministerio de Educación y Cultura.

Artículo 2. ${ }^{\circ}$ Se entiende como "Pasantía Educativa Laboral" la práctica profesional que realiza un estudiante para poner en ejercicio sus competencias y capacidades. Se concibe como el acto educativo regulado por el Ministerio de Educación y Cultura, que desarrolla el alumno en el ámbito de una empresa u organización privada legalmente constituida o en una entidad pública, con la finalidad de complementar el proceso de aprendizaje por medio de la aplicación práctica de la formación académica que recibe. Artículo 3. ${ }^{\circ}$ A los efectos de esta ley, son Pasantías Educativas Laborales obligatorias aquellas que forman parte del itinerario formativo prescripto en el currículum de la especialidad, y que constituyen un requisito para acceder al título correspondiente.

Fuente: Elaboración propia a partir de la CN (1992); Ley N 1.264/98; Ley Nº 4264 y la Ley N 5.636 
La Constitución Nacional de la República de Paraguay (1992) La Constitución Nacional expresa el derecho a la educación y la protección del/del niño/a.; como fuente de toda la base legal de la educación en Paraguay, y las leyes aplicadas: entre las que se tiene la Ley General de Educación $\mathrm{N}^{\circ}$ 1.264/98; En la sección IV se delinean las pautas legales sobre educación media. Entre tanto la Ley $\mathrm{N}^{\circ} 4264$ Pasantía Educativa Laboral, y la Ley $\mathrm{N}^{\circ} 5.636$ Pasantía Educativa Laboral Técnica Superior. Una descripción más precisa en la Tabla 1.

\subsection{EDUCACIÓN TÉCNICO - PROFESIONAL EN EL PARAGUAY}

La educación produce cambios en las personas, las economías y las sociedades, y a la vez está profundamente afectada por los resultados de estos cambios. En ese sentido, requiere ser cada vez más consciente de sí misma y de su entorno, manteniéndose en constante evolución, para ser relevante y valiosa, y contribuir al desarrollo sostenible.

En directa relación con la calidad de la educación, se advierte, el profundo grado de desconexión que existe entre la formación que ofrece el sistema educativo, y las competencias demandadas por el sector productivo. Dentro de las regiones emergentes, América Latina y el Caribe es la que mayores dificultades registra en relación a las calificaciones demandadas por el mercado, con un $36 \%$ de empresas del sector formal que declara tener problemas para encontrar una fuerza laboral adecuadamente formada. Este resultado contrasta con el $21 \%$ del promedio mundial y un 15\% en los países de la OCDE. Las empresas con procesos productivos intensivos en competencias son las que enfrentarían las mayores dificultades, en particular las pertenecientes a sectores con mayor grado de sofisticación, conectividad y complejidad dentro del conjunto de industrias transables (OECD, 2014). Esto resulta desafiante, considerando que estos sectores pueden ser claves para el proceso de transformación estructural de la región, cuya matriz productiva está centrada en industrias de poco valor agregado y baja productividad. OREALC/UNESCO (2016, p. 13)

La educación secundaria ha sido el espacio históricamente privilegiado para la implementación de la EFTP en la región. Como resultado, muchos países cuentan en el ciclo superior de este nivel con una oferta amplia de enseñanza técnica y profesional que ha prevalecido en el tiempo. Ese es el caso de Cuba, Chile, Ecuador y El Salvador, en los que la matrícula de esta educación supera el $40 \%$ respecto al total del alumnado.

En otros países, como Argentina, Colombia, Costa Rica y Paraguay la oferta de EFTP está en el orden del 20\%. (Contreras, 2015) citado por McOmish, E., \& Chakroun, B. \&.Barbieri, C. (2016), en OREALC/UNESCO (2016, p. 13)

Aunque menos extendida, la oferta de educación técnica en el ciclo inferior de la secundaria, continúa presente en la región. En los últimos años países como Paraguay, Uruguay y México han potenciado sus programas técnicos básicos a fin de disminuir la deserción en los primeros años de la secundaria, particularmente la de estudiantes de menor nivel socioeconómico o asentados en zonas rurales. Un componente importante de los programas de EFTP es el aprendizaje en los lugares de trabajo, el que puede tomar variadas formas tales como, pasantías, prácticas profesionales, formación dual, y contratos de aprendices.

En el nivel secundario, si bien se reconoce que las prácticas profesionales son fundamentales para validar los aprendizajes logrados en las escuelas y adquirir nuevos conocimientos y destrezas, no todos los estudiantes que acceden a la enseñanza técnica y profesional tienen experiencia práctica en empresas. En Argentina, por ejemplo, las prácticas profesionalizantes empiezan a implementar 
como estrategia formativa, curricular y obligatoria recién desde 2013. En otros países, como Chile y Ecuador, aun cuando estas prácticas son parte constitutiva de sus programas técnicos escolares, menos de la mitad de los estudiantes las realiza. En Paraguay, si bien la modalidad de Bachillerato Técnico incluye una pasantía curricular obligatoria de 240 horas como mínimo, a fin de ofrecer a los estudiantes una primera experiencia en el mundo del trabajo, no existen mecanismos de monitoreo que permitan asegurar su efectividad. McOmish, E., \& Chakroun, B. \&.Barbieri, C. (2016), en OREALC/UNESCO (2016, p. 15)

Otro aspecto clave para la calidad de la EFTP, es la preparación de profesores y formadores. Salvo algunas excepciones, esta es la mayor deuda que tiene la región con esta educación y que limita la aplicación de metodologías y tecnologías de enseñanza innovadoras.

En el Paraguay,

"no existen programas específicos de formación para docentes de EFTP, excepto algunos experimentos aislados en los sectores público y privado. Lo mismo ocurre en Costa Rica, donde la formación de los docentes de las escuelas técnicas del Ministerio de Educación Pública es principalmente académica, y no contempla instancias de aprendizaje en los puestos de trabajo. En este país, como sucede en muchos de la región, los requisitos para impartir clases en estas escuelas son distintos a los que impone el INA para la formación profesional, lo que dificulta que se compartan recursos para hacer frente a los escases de docente y formadores capacitados." McOmish, E., \& Chakroun, B. \&.Barbieri, C. (2016), en OREALC/UNESCO (2016, p. 22)

La EFTP como bien público La educación debe ser una misión compartida de la sociedad, lo que implica un proceso inclusivo en la formulación e implementación de políticas públicas.

La noción de bien público de la EFTP resalta su carácter colectivo, subrayando que:

"la relevancia que tiene en esta educación la consideración de todos los actores involucrados, los que cumplen roles particulares y que son insustituibles. En ese sentido, la EFTP requiere alianzas entre el Estado, la sociedad civil, y el sector privado, con la participación de las empresas. El rol del Estado es esencial como ente regulador y fijador de normas y estándares de calidad, particularmente cuando aumenta la participación del sector privado en la provisión de la EFTP.” McOmish, E., \& Chakroun, B. \&.Barbieri, C. (2016), en OREALC/UNESCO (2016, p. 27)

Gustavo Volpe, presidente de la UIP destacó la importancia de la formación de jóvenes para que éstos puedan acceder a fuentes de trabajo dignos y bien remunerados, "desde la UIP tomamos el desafío de formar en la educación técnica, las industrias son fuentes genuinas de trabajo y al contar con personal calificado, potenciamos los recursos y las empresas crecen". Situación por la que se encuentra la Educación ETP y la disponibilidad de competencias técnicas, el sector privado industrial enfrenta la necesidad de una mayor formación técnica de jóvenes, a fin de incorporar mano de obra calificada que favorezca al desarrollo del país.

Ante esta situación, la Unión Industrial Paraguaya UIP, a través del Instituto Técnico Superior del Centro Paraguayo de Productividad y Calidad (ITS CEPPROCAL), ofrece a jóvenes la posibilidad de estudiar carreras técnicas de mandos medios, con rápida salida laboral en el sector. Las ofertas de formación para cursar las carreras de Técnico Superior se encuentran: Mecatrónica Industrial, Ventas, Farmacia, Logística Integral, Administración, Recursos Humanos, Salud y Seguridad Ocupacional, Productividad y Calidad. (Volpe, 2018). 
Por otro lado, otro impacto no menos doloroso, El Foro Económico Mundial en su Índice de Competitividad Global 2016-2017, al Paraguay la ubica en el tercer lugar, luego de la corrupción y de la infraestructura inadecuada. En alusión al tema, el Departamento de Estrategias Comerciales e Integración, MH (2017), presenta cuáles son los factores más problemáticos para hacer negocios en Paraguay para 2016/2017.

Se destaca en primer lugar:

"La corrupción con un puntaje de 18,2; siendo levemente disminuido con respecto al año anterior $(18,7)$. En segundo se ubica la fuerza laboral inadecuadamente formada, la cual ha mejorado con una posición de 15,0 respecto al 18,7 del año anterior. En tercer lugar, se encuentra la provisión inadecuada de infraestructura $(14,5)$; seguida por la burocracia gubernamental inadecuada $(13,6)$ que ha tenido un retroceso con respecto al año anterior. En cuarta posición, mejorando con 10,5, frente al 8,2 anterior, se encuentra el acceso al financiamiento. No obstante, los factores en los que existe una mayor ventaja para hacer negocios en Paraguay son: regulaciones tributarias $(0,3)$, tasas de interés $(0,4)$ e inflación $(0,7)$. El Estado no tiene el papel de crear empleos, sino el de establecer las condiciones propicias para que el sector privado los genere. Una de ellas supone preparar a los jóvenes, a través de la educación pública, de tal modo que respondan a las demandas del mercado. Se puede concluir que, si bien se presentaron avances dentro de los componentes que corresponden a Paraguay, que lo llevaron a mejorar en una posición con respecto al año anterior, los mayores desafíos enfrentados por Paraguay continúan siendo el fortalecimiento de las instituciones; el fomento de la inversión para la provisión de infraestructura y conectividad debido a su condición de país en desarrollo sin litoral marítimo, hecho que impulsaría el comercio y la producción nacional. Otro importante desafío para Paraguay es el de mejorar la salud pública y la educación, en especial el fortalecer la educación terciaria y la capacitación de su fuerza laboral. Con respecto al mercado, es necesario mejorar la sofisticación empresarial; así como el mercado financiero". (Departamento de Estrategias Comerciales e Integración, 2017, p.9)

En ADN Digital (2017), publican que las industrias maquiladoras habilitaron nuevos puestos de empleo que se sumarán a los 13.000 ya existentes y ocupados. Esta vez fueron cubiertos 155 nuevos puestos, en su mayoría por gente joven. Enrique López Arce, director de Empleo del Ministerio del Trabajo, explicó que:

"las vacancias cubiertas son para operarios de fábricas, costureros industriales, técnicos en costuras con experiencia o junior, soldadura en general, técnicos en electrónica, torneros, chapistas, pintores y operadores de call center, y logramos acercar al postulante a las compañías que buscaban trabajadores y no estaban encontrando".

Considerando los antecedentes de formación ETP, de la disponibilidad y calidad del capital humano es menester trabajar con políticas públicas que den solución a corto plazo del dilema industrialización y mano obra técnica en el Paraguay. La premura surge no solo de la necesidad del desarrollo laboral, productivo y económico, sino también del desarrollo educativo y técnico del país. Pues mientras países de la región actualizan su sistema educativo e integran la educación al desarrollo del país, Paraguay permanece con ambigüedades en el campo de la educación técnico profesional. 


\section{NECESARIO FORTALECIMIENTO DEL SISTEMA DE EDUCACIÓN TÉCNICO PROFESIONAL EN EL PARAGUAY}

Habida cuenta de la gran necesidad de disponer de un sistema de formación técnico profesional, que, junto a las universidades, instruyan al mayor número posible de jóvenes en cualificaciones profesionales que facilite su acceso al mercado laboral. Es preciso, al mismo tiempo, contar de sistemas formativos específicos pensados para grupos vulnerables. Por tanto, el diseño y desarrollo de un sistema de Educación Técnico Profesional debe estar vinculado con el sistema educativo y con el sistema productivo adecuado a sus demandas laborales, que lo vuelve una condición sine qua non para reducir de manera sustantiva el trecho entre educación y empleo. El fortalecimiento de un sistema de Educación Técnico Profesional así pensado ha de velar por la presencia de expresas tipologías que desarrollen su validez y su capacidad de formalizar los objetivos deseados.

Para el logro efectivo de los objetivos deseados es menester contar con suficientes profesionales especializados tanto en el sistema educativo como en el sistema de formación técnico profesional. De igual forma contar la efectiva coordinación de ambos subsistemas en el desarrollo de sus funciones específicas. También lograr el acuerdo institución - empresa, instituciones públicas, ONGs, vinculación muy importante para lograr la sinergia y el desarrollo social.

No menos importante, ni se descarta, se augura la impronta que trae las Becas Carlos Antonio López (Becal) que marca un antes y un después en la historia de la educación superior en Paraguay. Desde 2015, más de 900 paraguayos ya fueron beneficiados con ayuda económica para cursar estudios de maestría y doctorado en varias de las 300 mejores universidades del mundo. Pero la brecha entre la formación técnica de mando medio sigue siendo deficiente para la industria que se instalan con mayor auge cada año.

\section{OBSERVACIONES EN MIRADA A LAS METAS EDUCATIVAS 2021}

Para realizar la Tabla 2; Tabla 3 y Tabla 4; nace de la idea de analizar el comportamiento en términos de avances y resultados logrados sobre el Proyecto Metas Educativas 2021 de la OEI, para ello se ha recurrido a las publicaciones de Miradas 2014, 2016, 2018, para poder recrear la evolución y los resultados cuantitativos por los países miembros; de la República del Paraguay en particular del Proyecto de referencia. Se presenta un avistamiento general de los resultados arrojados al 2015 de la meta alusiva analizando los aspectos más relevantes de realidad educativa en el Paraguay.

Tabla 2: Meta Específica 16: Mejorar y adaptar el diseño de la educación técnico-profesional de acuerdo con las demandas laborales.

\begin{tabular}{|l|c|c|c|c|c|}
\hline \multicolumn{2}{|l|}{ PARAGUAY: Nivel de logro: En 2015, entre el 20\% y el 70\% de los centros de formación } & \multicolumn{2}{c|}{$\begin{array}{c}\text { Año } 2015 \\
\text { CINE } 3\end{array}$} \\
técnico-profesional organiza las carreras en función de las competencias derivadas de la \\
demanda laboral, y entre el 50\% y el 100\% lo concreta en el 2021
\end{tabular}


5) cuyos currículos son diseñados teniendo en cuenta la demanda laboral

Fuente: Elaboración propia a partir de Miradas 2014, 2016, 2018 - Metas Educativas 2021

La tabla 2 nos evidencia que el 2014, que representantes paraguayas han reportado algunos datos importantes para la Mejora y adaptación del diseño de la educación técnico-profesional de acuerdo con las demandas laborales, del indicador 23 A; CINE 3 y CINE 5; en tanto para el indicador 23 $\mathrm{B}$ no se dispone de información.

La Tabla 3: el indicador 24 se ocupa de Mejorar y adaptar el diseño de la ETP de acuerdo con las demandas laborales, para el CINE 3 y CINE 5

Tabla 3: Meta Específica 16: Mejorar y adaptar el diseño de la ETP de acuerdo con las demandas laborales.

\begin{tabular}{|l|c|c|c|c|c|}
\hline $\begin{array}{l}\text { PARAGUAY: En 2015, entre el 30\% y el 70\% de los alumnos de ETP realiza } \\
\text { prácticas en empresas o instituciones laborales, y entre el 70\% y el 100\% lo hace } \\
\text { en 2021 }\end{array}$ & \multicolumn{2}{|c|}{$\begin{array}{c}\text { Año 2015 } \\
\text { CINE 3 } \\
\text { CINE 5 }\end{array}$} \\
\hline INDICADOR 24 & $\begin{array}{c}2014 \\
\text { CINE 3 }\end{array}$ & $\begin{array}{c}2016 \\
\text { CINE } \\
3\end{array}$ & $\begin{array}{c}2018 \\
\text { CINE } \\
3\end{array}$ & $30 \%$ & $70 \%$ \\
\hline $\begin{array}{l}\text { Porcentaje de alumnos de CINE 3 y } 5 \text { que realizan } \\
\text { prácticas formativas en empresas }\end{array}$ & $\mathrm{C}_{3}$ & $\mathrm{n} / \mathrm{d}$ & $\mathrm{n} / \mathrm{d}$ & $\mathrm{n} / \mathrm{d}$ & $\mathrm{n} / \mathrm{d}$ \\
& $\mathrm{C}_{5} \mathrm{n} / \mathrm{d}$ & & & & \\
& & & & \\
\hline
\end{tabular}

Fuente: Elaboración propia a partir de Miradas 2014, 2016, 2018 Metas Educativas 2021

En esta rúbrica del indicador 24; que se ocupa de Mejorar y adaptar el diseño de la ETP de acuerdo con las demandas laborales, para el CINE 3 y CINE 5. Solamente se conoce de resultados proporcionados del sector EFTP y del CINE 5 sin datos.

Tabla 4; busca información de titulaciones entre una edad etaria de 18 a 21 años de edad de la población procedentes de la educación técnico profesional de nivel medio superior (CINE 3)

Tabla 4: Meta Específica 17: Aumentar y mejorar los niveles de inserción laboral en el sector formal de los jóvenes egresados de la ETP

\begin{tabular}{|l|l|c|c|c|c|c|}
\hline $\begin{array}{l}\text { PARAGUAY: En 2015, entre el 30\% y el 60\% de los egresados de la ETP } \\
\text { consigue una inserción laboral acorde con la formación obtenida, y entre } \\
\text { el 50\% y el 75\% lo logra en 2021 }\end{array}$ & \multicolumn{2}{|c|}{$\begin{array}{c}\text { Año 2015 } \\
\text { CINE 3 }\end{array}$} \\
\hline INDICADOR 25 & $\begin{array}{c}2014 \\
\text { CINE } \\
3\end{array}$ & $\begin{array}{c}2016 \\
\text { CINE } \\
3\end{array}$ & $\begin{array}{c}2018 \\
\text { CINE } \\
3\end{array}$ & $30 \%$ & $70 \%$ \\
\hline $\begin{array}{l}\text { Porcentaje de titulados de 18 a 21 años de edad, } \\
\text { procedentes de la educación técnico profesional de nivel } \\
\text { medio superior (cine 3), que acceden al empleo en } \\
\text { puestos afines a su capacitación después de terminar sus } \\
\text { estudios. }\end{array}$ & $\mathrm{n} / \mathrm{d}$ & $\mathrm{n} / \mathrm{d}$ & $\mathrm{n} / \mathrm{d}$ & $\mathrm{n} / \mathrm{d}$ & $\mathrm{n} / \mathrm{d}$ \\
\end{tabular}

Fuente: Elaboración propia a partir de Miradas 2014, 2016, 2018 Metas Educativas 2021 
En este informe de 2016, Se remite a lo recogido en el documento anterior de seguimiento de las Metas Miradas 2014.

En este caso en particular el indicador 25; no ofrece información alguna en relación a titulaciones entre una edad etaria de 18 a 21 años de edad de la población procedentes de la educación técnico profesional de nivel medio superior (CINE 3)

Con las informaciones obtenidas y los datos, se evidencia que con cada gobierno de turno cambian los programas y proyectos y a ninguna de ella realizan seguimientos, por tanto, no se dispone de datos certeros de las condiciones actuales de los programas la mayoría de ellas vigentes. Para todos los casos urge una revisión a profundidad para obtener resultados inequívocos a fin de tomar las decisiones preventivas o correctivas a los mismos. Para METAS EDUCATIVAS 2021se han elaborado indicadores a fin observar la evolución de los resultados, en un tiempo determinado. En ese contexto se detalla algunos: Indicador 23A, el Porcentaje de carreras técnico-profesionales de nivel educativo medio superior y terciario (Niveles CINE 3 y 5) cuyos currículos son diseñados por competencias.

Paraguay reporta información en el 2014 en CINE 3 100\% y CINE 5 70\%. En cuanto al indicador 23B Porcentaje de carreras técnico-profesionales de nivel educativo medio superior y terciario (Niveles CINE 3 y 5) cuyos currículos son diseñados teniendo en cuenta la demanda laboral, el Paraguay no reportó información. El indicador 24 Porcentaje de alumnos de CINE 3 y CINE 5 que realizan prácticas formativas en empresas; CINE 3 logró $47 \%$ en tanto CINE $5 \mathrm{n} / \mathrm{d}$; y el indicador 25 Porcentaje de titulados de 18 a 21 años de edad, procedentes de la educación técnico profesional de nivel medio superior (cine 3), que acceden al empleo en puestos afines a su capacitación después de terminar sus estudios. El Paraguay no arrojó información alguna. Se puede valorar que a partir de estos resultados la cartera de educación se abocó en elaborar un el Marco Legal que coadyuve al aseguramiento de la EFTP que se han enunciado desde el seno de la CN (1992); estas leyes hoy disponibles para colegir en las acciones iniciadas, la Ley $\mathrm{N}^{\circ} 1.264 / 98$; Ley $\mathrm{N}^{\circ} 4264$ y la Ley $\mathrm{N}^{\circ} 5.636$.

Los retos para una educación técnica para el siglo XXI son innumerables, debe de crearse los mecanismos para que los sectores sociales más vulnerables puedan expresar sus demandas legítimas por educación, propender que los recursos para educación sean más apropiados para que todos, jóvenes y adultos tengan oportunidades de capacitación y así construir un futuro más esperanzador para él y su familia.

\section{CONSIDERACIONES DE LA EVOLUCIÓN DE METAS EDUCATIVAS 2021}

En el informe Miradas 2011 ya se señalaba cómo el avance tecnológico de los últimos tiempos, unido al alto grado de especialización necesario para determinados sectores productivos, determina que en el mercado laboral adquiera una escala progresiva de mejora de las capacidades profesionales en varios campos laborales. Por tanto, se debe trabajar cada vez más en las competitividades que deben desplegar, con una configuración más adecuada, abierta al futuro y vinculada a las posibilidades de aprendizaje para toda la vida. Condición se está desarrollando en todos los países, e Iberoamérica sigue los mismos pasos.

Para el Paraguay en estas circunstancias, con los resultados e arrojan los informes de las publicaciones de distintas ediciones de Miradas Metas Educativas 2021, no son nada alentadores por el nivel de logro acordado para 2015 y 2021, a estas alturas del proyecto a tres años de concluirse 
se halla muy lejos de un avance mínimamente satisfactorio; solo con el deber de replantear los indicadores o indagar nuevas formas de ejecutar y lograr algún resultado más deseable.

\title{
7. INVERSIÓN Y GASTO PÚBLICO EN EDUCACIÓN, TOTAL (\% DEL PIB) EN EL PARAGUAY
}

La inversión pública en educación es calificada un componente imprescindible para el crecimiento económico y en el desarrollo social de cualquier país, al otorgar a su mano de obra de la capacidad técnica para lograr un nivel de rendimiento productivo. Empero, el Gasto en Educación Per Cápita, Paraguay se encuentra muy por debajo del promedio regional siendo el país con menor inversión educativa per cápita. Es bueno echar de ver que el Gasto Per cápita se trata del gasto total en educación dividido entre todos los habitantes, independientemente de que sean estudiantes o no y de la edad que tengan.

Tabla 5: Inversión en educación (2002-2014p) (\% del PIB)

\begin{tabular}{|l|c|c|c|c|c|c|c|c|c|c|c|c|c|}
\hline Educación y cultura & 2002 & 2003 & 2004 & 2005 & 2006 & 2007 & 2008 & 2009 & 2010 & 2011 & 2012 & 2013 & $2014 p$ \\
\hline Educación elemental & 0,00 & 0,00 & 1,61 & 1,69 & 1,71 & 1,63 & 1,74 & 1,90 & 1,73 & 1,75 & 1,99 & 1,82 & 1,74 \\
\hline Educación media y técnica & 0,05 & 0,02 & 0,73 & 0,74 & 0,77 & 0,73 & 0,77 & 0,88 & 0,80 & 0,81 & 0,85 & 0,75 & 0,66 \\
\hline $\begin{array}{l}\text { Educación superiory } \\
\text { universitaria }\end{array}$ & 0,44 & 0,44 & 0,47 & 0,55 & 0,59 & 0,68 & 1,30 & 1,61 & 1,42 & 1,45 & 1,73 & 1,77 & 1,81 \\
\hline Cultura & 0,00 & 0,00 & 0,00 & 0,02 & 0,02 & 0,02 & 0,03 & 0,03 & 0,03 & 0,07 & 0,05 & 0,04 & 0,04 \\
\hline $\begin{array}{l}\text { Deportes y recreación } \\
\text { Sin discriminar }\end{array}$ & 0,00 & 0,00 & 0,00 & 0,00 & 0,00 & 0,01 & 0,00 & 0,00 & 0,01 & 0,02 & 0,04 & 0,04 & 0,04 \\
\hline Total & $\mathbf{3 , 2 5}$ & $\mathbf{2 , 6 6}$ & 0,78 & 0,78 & 0,88 & 0,93 & 0,25 & 0,32 & 0,32 & 0,30 & 0,35 & 0,39 & 0,48 \\
\hline
\end{tabular}

Fuente: Datos del MH y el BCP. (Velaztiqui, 2015).

Velaztiqui (2015), señala que los gastos correspondientes a la inversión en educación media y técnica en el Paraguay incluyen:

\begin{abstract}
"aquellos en las escuelas de enseñanza media y técnica, escuelas medias artísticas, técnicas y profesionales, tanto en los primeros años (ciclos básicos) como en los restantes. Incluye la enseñanza que se imparte en establecimientos de protección de menores. La educación superior y universitaria incluye las erogaciones correspondientes a la administración y gestión de los programas de enseñanza superior y formación de profesionales para obtener certificado, diploma o título universitario que sirva para el ejercicio de empleos especializados, de la carrera, y los programas de actualización de profesionales y especialistas. Incluye la instrucción y formación impartida en academias y escuelas de instrucción militar con alto contenido académico. La inversión en cultura incluye los gastos destinados a las manifestaciones intelectuales y espirituales de la comunidad, difusión de conocimientos por radio, televisión u otros medios de comunicación, cursos de extensión y espectáculos públicos culturales, museos, bibliotecas, monumentos y lugares históricos. No comprende las actividades específicamente dirigidas a brindar educación o a proporcionar entretenimiento. Por último, la inversión en deporte y recreación incluye los gastos destinados a la promoción de las actividades deportivas, cultura física, recreación y esparcimiento de la población. La inversión en educación elemental y en educación superior y universitaria representan las principales erogaciones de la inversión en educación, ambos con un promedio del 1,8\% del PIB en los dos últimos años $(4,9$ billones en total en 2014); y en el tercer lugar, se encuentra la inversión en educación media y técnica con solo el 0,7\% del PIB del último año. Tabla 5. Se puede observar, asimismo, durante el periodo que la inversión en educación elemental y media y técnica presentan casi el mismo promedio cercano al $1,6 \%$ y $0,7 \%$ del PIB anual respectivamente, mientras que la inversión en educación superior y universitaria presenta una tendencia creciente entre 2002 y 2014". (pp. 38-39).
\end{abstract}




\subsection{GASTO SOCIAL EN LA REGIÓN}

Tabla 6: Gasto social en la Región

\begin{tabular}{|c|c|c|c|c|c|}
\hline \multicolumn{6}{|c|}{ COMPARATIVO SOBRE GASTO SOCIAL EN LA REGIÓN } \\
\hline \multicolumn{6}{|c|}{ *Datos comparativos 2015 - En porcentaje del PIB } \\
\hline Pais & Educación & Protec. Social & Salud & Cultura & Gasto en \% PIB \\
\hline Chile & $4,69 \%$ & $6,28 \%$ & $4,41 \%$ & $0,19 \%$ & $16,03 \%$ \\
\hline 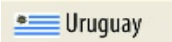 & $4,44 \%$ & $6,77 \%$ & $3,00 \%$ & $0,18 \%$ & $14,91 \%$ \\
\hline - Argentina & $6,63 \%$ & $10,85 \%$ & $7,10 \%$ & $0,25 \%$ & $14,70 \%$ \\
\hline - Brasil & $5,55 \%$ & $10,26 \%$ & $5,06 \%$ & $0,26 \%$ & $12,79 \%$ \\
\hline Bolivia & $5,85 \%$ & $4,38 \%$ & $1,91 \%$ & $1,07 \%$ & $12,61 \%$ \\
\hline Paraguay & $4,28 \%$ & $4,96 \%$ & $2,67 \%$ & $0,10 \%$ & $12,01 \%$ \\
\hline
\end{tabular}

Fuente: Base de datos de inversión social en América Latina y el Caribe de la Cepal.

La Tabla 6; Gasto social en la Región, la CEPAL realiza una comparación en la región en términos porcentuales del PIB, el estudio toma datos desde el año 2000 al 2015; en el último año de referencia se indica que el gasto social de Paraguay al 2015 representaba un 12,01\% de su PIB. Esta cifra ubica al Paraguay en el último lugar, al comparar con los porcentajes de países vecinos como Chile (16,03\%), Uruguay (14,91\%), Argentina (14,70\%), Brasil (12,79\%) y Bolivia (12,61\%) de su PIB. (2017) $14,46 \%$ de su producto interno bruto (PIB) al gasto social.

Es evidente que toda la pobreza radica en la falta de educación del pueblo, con los datos a la vista es todas luces del desinterés histórico de los gobiernos de turnos en propender a un mejor recurso para el área de educación. La educación no se puede mirar como un gasto, siempre será una gran inversión. Un país educado es sinónimo de salud y seguridad.

Gráfico 1 y Tabla 7, presentan un panorama de los datos comparativos entre, lo que organismos como la UNESCO y fuentes nacionales disponen, que hace alusión al gasto público en educación, tanto en términos porcentuales del PIB y en moneda extranjera (dólares), de esta forma contrastar los resultados y confirmar que en el Paraguay, las necesidades en educación son históricas y presentes, y no alcanzan satisfacerlas sin una mayor inversión pública, siendo uno de los países de la región con menor inversión en educación, de esta manera se están socavando contra que pueda revertir en el binomio desarrollo social y desarrollo económico. Indiscutiblemente que en la educación descansan las bases para el desarrollo de las personas y de un país, no existe otro contexto ni se conoce otra receta. 


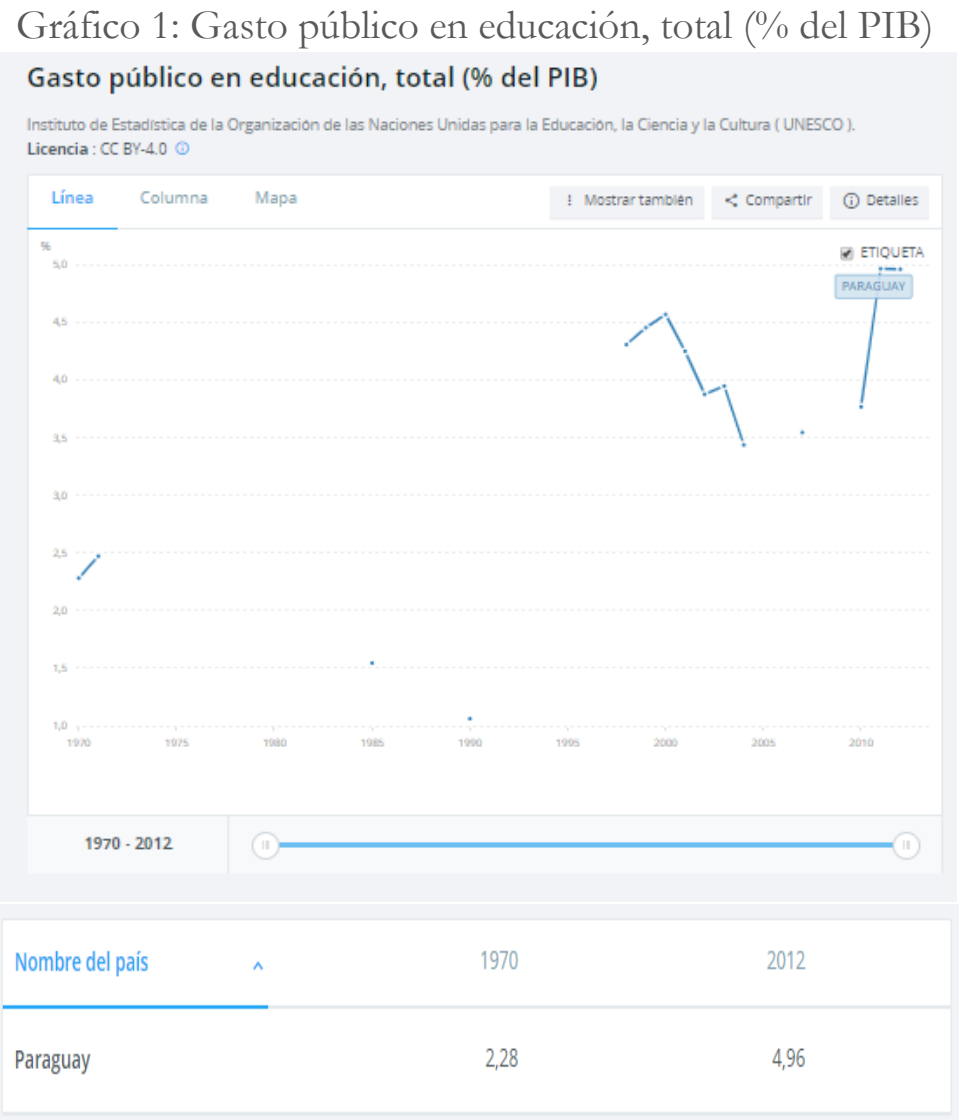

Cabe destacar, que a falta de informaciones con datos estadísticos del avance del Proyecto Metas Educativas 2021, de la cartera de educación del Paraguay se vuelve complejo trabajar. Si bien existe mucha información de otros programas en ejecución pero sin los datos que acompañe el avance, y la falta de seguimientos de cada uno de los programas y proyectos aprobados con cada cambio de gobierno.

Tabla 7: Gasto público Educación (En dólares)

\begin{tabular}{|c|c|c|c|c|}
\hline Años & $\begin{array}{c}\text { Gasto en } \\
\text { Educación } \\
\text { (Millones de } \\
\text { \$) }\end{array}$ & $\begin{array}{c}\text { Gasto en } \\
\text { Educación } \\
\text { (\%Gasto Pub. } \\
\text { Total) }\end{array}$ & $\begin{array}{c}\text { Gasto en } \\
\text { Educación } \\
\text { (\%PIB) }\end{array}$ & $\begin{array}{c}\text { Gasto en } \\
\text { Educación Per } \\
\text { Cápita (En \$) }\end{array}$ \\
\hline 2002 & 246 & 18,8 & 3,9 & 44 \\
\hline 2003 & 260 & 20,9 & 4,0 & 46 \\
\hline 2004 & 275 & 18,2 & 3,4 & 48 \\
\hline 2007 & 489 & 19,3 & 3,6 & 80 \\
\hline 2010 & 653 & 15,7 & 3,8 & 101 \\
\hline 2011 & 931 & 18,7 & 5,0 & 142 \\
\hline 2012 & 1.015 & 16,1 & 5,0 & 152 \\
\hline $2013^{*}$ & 1.296 & 16,4 & 4,5 & 193 \\
\hline $2014^{*}$ & 1.288 & 14,9 & 4,2 & 189 \\
\hline $2015^{*}$ & 1.335 & 16,4 & 4,4 & 193 \\
\hline
\end{tabular}

*Datos estimados en base al Presupuesto General de la Nación de cada año y

datos del Banco Central del Paraguay.

Fuente: Banco Mundial. Indicadores de Desarrollo Mundial.

http://data.worldbank.org 


\section{CONCLUSIONES}

El objetivo del trabajo es contribuir a la reflexión sobre el nivel de logro que han alcanzado los Estados firmantes del proyecto en torno a las 11 metas educativas propuestas a tres años vista de la finalización del periodo de su desarrollo. Que por cierto los estados firmantes han logrado avances importantes en torno a las 11 metas educativas. Este trabajo se centró más en analizar el avance logrado en el Paraguay del objetivo de la Sexta Metas Educativas 2021, la de Favorecer la conexión entre la educación y el empleo a través de la educación técnico-profesional (ETP). Para el desarrollo del trabajo se recurrió a la revisión de publicaciones de la UNESCO con relación a Metas Educativas 2021 de sus avances y retrocesos y las publicaciones que las instituciones rectoras de la educación paraguaya ofrecen, y otras disponibles en los medios.

La inversión en la educación paraguaya es exigua, causal de expulsión del sistema educativo que se traduce en pocos años de escolaridad de un sector importante de la población, y por sobre todo la educación técnico profesional. Metas Educativas 2021 como otros programas han fracasado por la falta de planificación estratégica en el país. Una Planificación Estratégica bien acompañada con un Plan de Desarrollo podría recuperar aprendizajes y capacidades para las acciones futuras en los discursos y cumplimientos de las políticas educativas. Un aspecto importante evidenciado es la iniciativa privada en la formación técnico profesional de la población, a raíz del déficit de mano de obra calificada demandada por las industrias asentadas en el país; iniciativa que permite una formación técnica que responda a las necesidades y demandas vigentes y proyectadas del sector productivo, facilitando así oportunidades reales de inserción laboral a las personas, que en contrapartida se logra el bienestar de las personas en la economía y la competitividad del país; con una mejor calidad de la educación técnico profesional acorde con las demandas del sector. Esta iniciativa responde a la realidad observada por el sector privado, logrando un impacto de desarrollo económico y humano en la población, reduciendo la brecha social, aumentando la empleabilidad y la competitividad en el país.

La educación es un derecho humano y bien público que permite a las personas ejerce $\mathrm{r}$ los otros derechos humanos (UNESCO/OREALC, 2007). Para concretarse el Derecho a la Educación de calidad para todos es preciso que el estado paraguayo realice una mayor inversión en el fortalecimiento del sector educativo, para el cumplimiento de las metas y el aseguramiento de la calidad educativa. La educación técnica se exhibe como el principal medio para aumentar la capacidad y creatividad de jóvenes y adultos, y su rápida inserción laboral con especialidades técnicas que hoy, más que nunca, demanda el país. La Educación Técnica Profesional es una estrategia elemental por su gran vinculación con el Sector socio - productivo, para el desarrollo de la población de una Nación; la educación, producción, profesiones y economía son aspectos que se articulan para que las sociedades puedan desarrollarse en beneficios de calidad de vida y suscitar el desarrollo humano y social.

Sucintamente, la educación contribuye al desarrollo humano y social, sin desarrollo social no es compatible un desarrollo económico, Paraguay debe luchar por una sociedad más justa, más productiva y con mayor equidad. El bien común que torna más libre a los seres humanos. 


\section{BIBLIOGRAFÍA}

Baelo, R., \& Fernández, M. y. (2018). Hacia una sociedad 4.0: Efectividad de las medidas educativas impulsadas en Castilla y León para. Castilla y León: Universidad de León. Obtenido de https://ceitriangular.org/wp-content/uploads/2018/11/MedidasEducativasSTEM.pdf

Comisión Económica para América Latina y el Caribe (CEPAL). (14 de junio de 2 017). Base de datos de inversión social en América Latina y el Caribe. Obtenido de Naciones Unidas: https://www.cepal.org/es/noticias/ya-esta-disponible-nueva-base-datos-inversion-socialamerica-latina-caribe.

Del Giorgio Solfa, F., Sierra, M., Vescio, M. V., \& Fernández Zocco, L. (febrero de 2018). La educación técnico-profesional en los procesos de desarrollo latinoamericanos. Reflexión Académica en Diseño y Comunicación NơXXIII, 33 (Año XIX), 188 a 198.

Elías, R. y. (2014). "Revisión Nacional 2015 de la Educación para Todos: Paraguay. Asunción: MEC.

Elías, R. (2014). Análisis de la reforma educativa en Paraguay: discursos, prácticas y resultados. Buenos Aires: CLACSO.

Fox, V. (2005). Análisis documental de contenido: principios y prácticas. Buenos Aires: Alfagrama.

Gómez, C., \& Sosa, T. (1998). Estado de avance de la reforma educativa de la formación técnico profesional en Paraguay. Educación y Trabajo (5). Obtenido de https://www.oei.es/historico/oeivirt/fp/cuad05a05.htm

Integración, D. d. (2017). Reporte de Competitividad Global 2016 -2017: Análisis sobre los resultados para la República del Paraguay. Asunción: Dirección de Integración-MH.

Jacinto, C. \&. (2009). Enfoques de Programas para la Inclusión laboral de los jóvenes pobres: lo institucional como soporte subjetivo. Última Década, 67-92.

Jacinto, C. (2009). Iniciativas recientes sobre formación para el trabajo en la educación secundaria general. Tendencias en Foco, RedEtis-IIPE-UNESCO, 10, 1-8. Obtenido de http://www.redetis.iipe-ides.org.ar

McOmish, E., \& Chakroun, B. \&.Barbieri, C. (2016). La enseñanza y formación técnico profesional en América Latina y el Caribe. Una perspectiva regional hacia 2030. Santiago: OREALC/UNESCO.

MEC, M. d. (2011). Plan de Mejoramiento de la Educación Térnica y Profesional en el Paraguay 2011-2013. Asunción: OEI. Obtenido de https://www.mec.gov.py/cms_v2/adjuntos/1968

Ministerio de Educación y Cultura MEC (2011). LEY N 4264 Pasantía Educativa Laboral. Asunción: Congreso de la Nación.

Ministerio de Educación y Cultura MEC. (2014). Informe Nacional Paraguay Educación para todos 20002015. Asunción: UNESCO. Obtenido de http://unesdoc.unesco.org/images/0023/002300/230036S.pdf 
Ministerio de Educación y Cultura MEC (2016). LEY N 5.636 Pasantía Educativa Laboral Técnica Superior. Asunción: Congreso de la Nación.

Molinas, J., \& Elías, R. y. (2004). Molinas, José; Elías, Rodolfo y Vera, Mirna 2004 Estudio y Análisis del Sector Educativo. Asunción: Instituto Desarrollo, JICA.

OEI, O. d. (2013). "Metas educativas 2021: Desafíos y oportunidades. Informe Siteal 2010. Buenos Aires: OEI. Obtenido de http://www.siteal.iipeoei.org/informe_2010

OREALC/UNESCO (2016). La enseñanza y formación técnico profesional en América Latina y el Caribe. Una perspectiva regional hacia 2030. Santiago - UNESCO

Organización de Estados Iberoamericanos para la Educación, la Ciencia y la Cultura OEI. (2014). Miradas sobre la Educación en Iberoamérica. Avances en las Metas Educativas 2021 (ISBN: 978-847666-206-9 ed.). Madrid: OEI.

Paraguay, P. d. (2011). Plan Nacional de mejoramiento de la educación técnica y profesional en el Paraguay 2011-2013. Asunción: Organización de Estados Americanos - OEI.

Paraguay, R. d. (1992). Constitución de la Republica de Paraguay. Asunción: Congreso de la Nación

Paraguay, R. d. (1998). Ley General de Educación N 1.264. Asunción: Congreso de la Nación.

SITEAL, S. d. (2010). El Informe sobre tendencias sociales y educativas en América Latina. METAS EDUCATIVAS 2021: DESAFÍOS Y OPORTUNIDADES. BUENOS AIRES: UNESCO.

Tedesco, J. (julio-agosto de 2016). Foro abierto OGE. Recuperado el 25 de octubre de 2018, de La educación en Latinoamérica. Transformaciones y futuro: https://www.ort.edu.uy/ie/articulos/la-educacion-en-latinoamerica.pdf

Velaztiqui, J. (2015). Evolución de la Inversión Pública Social en Paraguay y los Objetivos del Milenio (2002-2014). Observatorio Fiscal - CADEP, 38-39.

Vickery, B. (1970). Techniques of information retrieval. Londres: Butterworths.

Vidales Calderón, P. (2013). Un México de Emprendedores: El reto de la educación. México: Instituto Mexicano de Ejecutivos de Finanzas.

Volpe, \& G. (3 de enero de 2018). Unión Industrial Paraguaya. doi:http:/ / www.uip.org.py/detalles.php?id=53 


\section{SOBRE EL AUTOR}

\section{Daxi Duarte de García}

Egresada con licenciatura en Administración de Empresas; Ex. Decana y docente de la Facultad de Ciencias Contables, Económicas y Administrativas de la Universidad Católica "Nuestra Señora de la Asunción"; República del Paraguay; con 22 años de docencia en la Educación Superior, actualmente Docente y Director de Cooperación y Relaciones Interinstitucionales de la Universidad Nacional de Villarrica del Espíritu Santo - UNVES. Máster en Educación: Planificación, Innovación y Gestión de la Práctica Educativa por la Universidad de Alcalá de Madrid; Máster Oficial en Bioética y Doctor en Investigación y Desarrollo por la Universidad Católica "San Vicente Mártir" de Valencia - España. Otros, Formación y Actualización en Procesos Regionales de Evaluación y Acreditación de la Calidad de la Educación Superior - San José - Costa Rica. IV Programa de Formación de Formadores en Responsabilidad Social Empresarial. UBA - Buenos Aires

Contact information: daxiduartevera@gmail.com

Universidad Nacional de Villarrica del Espiritu Santo - UNVES, Av. Boulevard Río Apa y Tapé Pytá, +595 54144535 